\title{
Resolved-sideband and cryogenic cooling of an optomechanical resonator
}

\author{
Young-Shin Park and Hailin Wang *
}

Cooling a mechanical oscillator to its quantum ground state enables the exploration of the quantum nature and the quantum-classical boundary of an otherwise classical system $^{1-7}$. In analogy to laser cooling of trapped ions ${ }^{8}$, ground-state cooling of an optomechanical system can in principle be achieved by radiation-pressure cooling in the resolved-sideband limit where the cavity photon lifetime far exceeds the mechanical oscillation period ${ }^{9-11}$. Here, we report the experimental demonstration of an optomechanical system that combines both resolved-sideband and cryogenic cooling. Mechanical oscillations of a deformed silica microsphere are coupled to optical whispering-gallery modes that can be excited through free-space evanescent coupling ${ }^{12,13}$. By precooling the system to $1.4 \mathrm{~K}$, a final average phonon occupation as low as 37 quanta, limited by ultrasonic attenuation in silica, is achieved. With diminishing ultrasonic attenuation, we anticipate that the ground-state cooling can be achieved when the resonator is precooled to a few hundred millikelvin in a ${ }^{3} \mathrm{He}$ cryostat.

In an optomechanical system, mechanical vibrations induce changes in the intracavity field as well as in the cavity-resonance frequency. Dynamical backaction of the radiation pressure can either damp or amplify the mechanical motion, depending on the laser detuning ${ }^{14,15}$. Radiation-pressure cooling as well as activeoptical-feedback cooling has been demonstrated in a variety of optomechanical systems ${ }^{16-22}$. Resolved-sideband cooling has also been realized recently in a toroidal silica resonator ${ }^{11}$. There are, however, considerable challenges in combining resolved-sideband cooling with cryogenic precooling for most optomechanical resonators ${ }^{23}$. The lowest average phonon occupation achieved in optomechanical resonators thus far remains above 1,000 quanta. In comparison, electromechanical systems can be cryogenically cooled to temperatures in the millikelvin range in a dilution refrigerator enabling an average phonon occupation as low as 25 (ref. 24). Dynamical backaction cooling in these systems has also been demonstrated recently ${ }^{24,25}$.

We have developed and explored the use of deformed silica microspheres as a special type of optomechanical resonator which are convenient for experimental studies in a cryogenic environment. Unlike whispering-gallery modes (WGMs) in conventional symmetric optical resonators such as spherical or toroidal resonators $^{26,27}$, WGMs in deformed non-axisymmetric microspheres can be effectively excited in free space through a directional evanescent tunnelling process. In these deformed resonators, the angle of incidence is no longer conserved. For WGMs near the equatorial plane, the angle of incidence becomes closest to the critical angle in regions $45^{\circ}$ away from either the long or short axis. At relatively small deformation, directional evanescent escape of WGMs can take place in these regions because the evanescent decay length as well as the evanescent tunnelling rate increases exponentially when the angle of incidence approaches

a

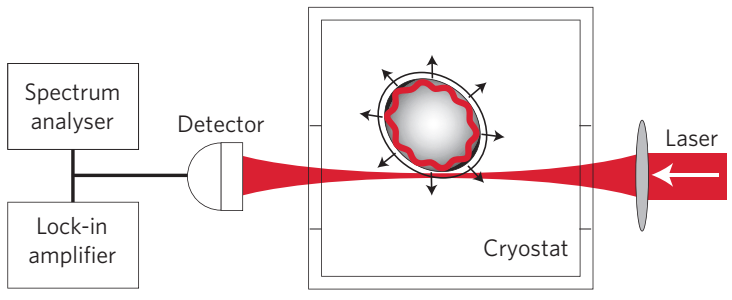

b

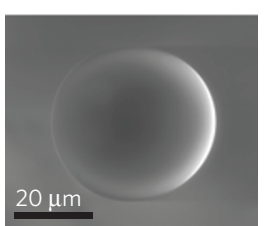

c

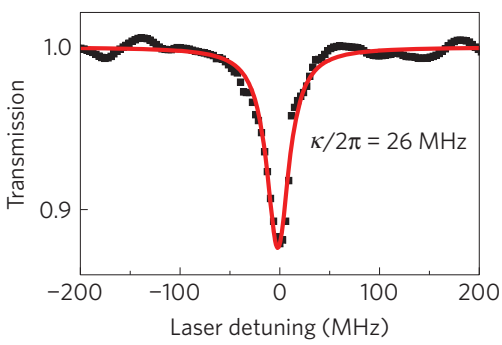

Figure 1 | Free-space coupling of WGMs. a, Schematic diagram of the experimental set-up for radiation-pressure cooling through free-space evanescent excitation of WGMs in a deformed silica microsphere. b, Scanning electron micrograph of a silica microsphere with deformation of $4.7 \%$, taken on the side opposite to the attached fibre stem. c, Optical transmission spectrum, obtained with free-space excitation, of a WGM resonance near $\lambda=800 \mathrm{~nm}$ for a microsphere with $d=30 \mu \mathrm{m}$ and deformation $<2 \%$. The solid line is a Lorentzian fit.

the critical angle of incidence ${ }^{12}$. WGMs can thus be excited in free space by focusing a laser beam to these regions, with the focal point just outside the sphere surface, as shown schematically in Fig. 1a (ref. 13). Figure 1b shows a scanning electron micrograph of a deformed silica microsphere. Figure 1c shows a WGM transmission resonance obtained through free-space excitation for a silica microsphere with a diameter $d=30 \mu \mathrm{m}$ and deformation less than $2 \%$. The transmission resonance features a cavity linewidth $\kappa / 2 \pi=26 \mathrm{MHz}$, corresponding to an optical quality factor, $Q \sim 1.4 \times 10^{7}$.

The experimental configuration for free-space excitation of WGMs can be used simultaneously for homodyne detection of mechanical vibrations of the optomechanical resonator. For light circulating in a WGM, mechanical vibrations including Brownian motion induce a phase shift, which is proportional to the mechanical displacement. Optical interferometric measurements of the induced phase shift provide a highly sensitive measure of the mechanical displacement ${ }^{28}$. In the free-space excitation configuration shown in Fig. 1a, the part of the excitation laser beam that is not coupled to WGMs provides a local oscillator for the homodyne interferometric detection of the mechanical displacement. 
$\mathbf{a}$

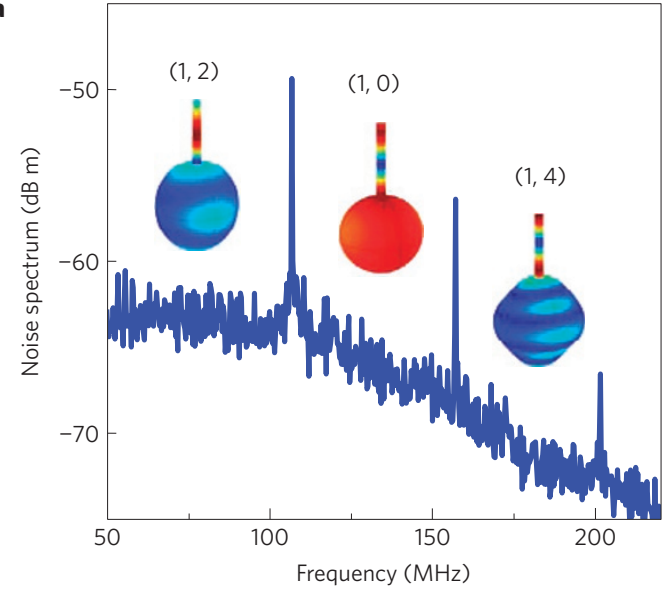

b

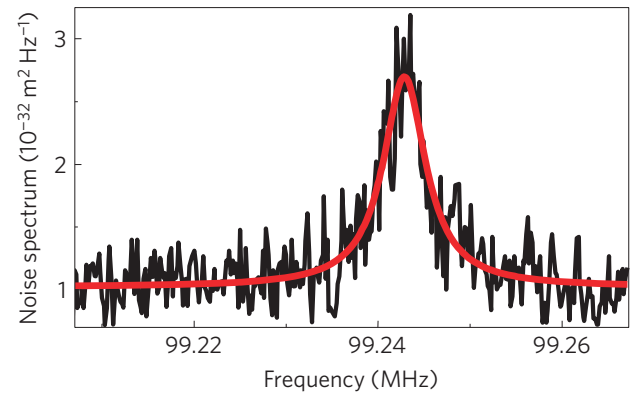

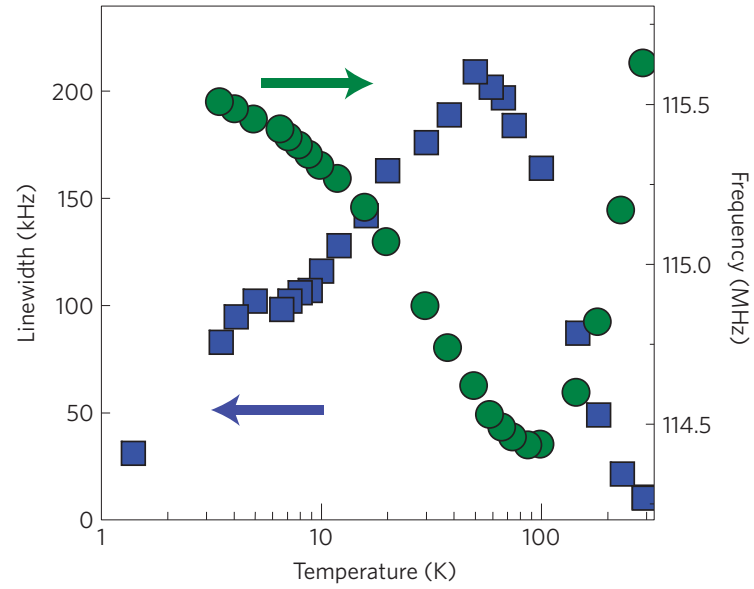

d

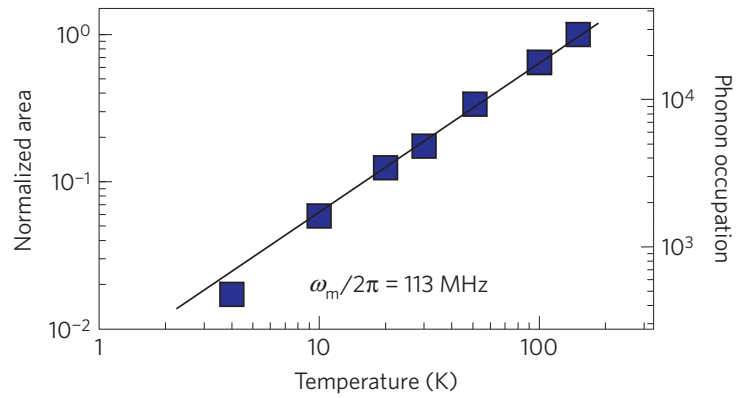

Figure 2 | Mechanical modes and cryogenic cooling. a, Displacement power spectrum showing the three lowest optically active mechanical modes of a deformed microsphere with $d=30 \mu \mathrm{m}$. The insets show the corresponding calculated spatial displacement patterns. $\mathbf{b}$, Displacement power spectrum of the $(1,2)$ mode in a deformed microsphere with $d=32 \mu \mathrm{m}$ obtained in vacuum and at room temperature. $A$ Lorentzian fit (red line) indicates $Q_{m}=18,000$. c, Temperature dependence of $\omega_{\mathrm{m}} / 2 \pi$ and $\gamma_{\mathrm{m}} / 2 \pi$ for the $(1,2)$ mode. $\mathbf{d}$, Spectrally integrated area of the $(1,2)$ mode in the displacement power spectrum as a function of $T_{\text {bath. }}$. The area is normalized to that obtained at $T_{\text {bath }}=150 \mathrm{~K}$. The solid line is the calculated area assuming that the mechanical oscillator is in thermal equilibrium with the bath.

Figure 2a shows the displacement power spectrum obtained with homodyne detection for a deformed silica microsphere with $d=30 \mu \mathrm{m}$. Three optically active mechanical breathing modes are observed. The insets in Fig. $2 \mathrm{a}$ also show the spatial displacement patterns calculated with finite-element analysis for the three mechanical modes, with mode number $(n, l)=(1,2),(1,0)$ and $(1,4)$, where $n$ and $l$ are the radial and angular mode numbers, respectively. The mechanical oscillation frequencies obtained in Fig. 2a, as well as their dependence on the sphere size, agree well with the theoretic calculation. The numerical calculation also yields an effective mass of $m_{\text {eff }}=41 \mathrm{ng}$ for the $(1,2)$ mode, which is in excellent agreement with the effective mass determined from a separate measurement using a phase-modulated laser beam (see Supplementary Information). The mechanical quality factor of a microsphere is in part limited by the damping induced by the attached fibre stem. With a small stem (less than $1 / 10$ of the sphere diameter) and a small deformation $(<2 \%)$, mechanical quality factors as high as $Q_{\mathrm{m}} \sim 10,000$ can be routinely achieved. $Q_{\mathrm{m}}$ as high as 18,000 has been observed in vacuum and at room temperature as shown in Fig. $2 \mathrm{~b}$, where the displacement, $x$, is derived from the equipartition theorem, $m_{\mathrm{eff}} \omega_{\mathrm{m}}^{2}\left\langle x^{2}\right\rangle=k_{\mathrm{B}} T$, with $\omega_{\mathrm{m}} / 2 \pi$ being the mechanical vibration frequency.

Figure $2 \mathrm{c}$ shows the temperature dependence of both intrinsic mechanical linewidth $\gamma_{\mathrm{m}} / 2 \pi$ and vibration frequency $\omega_{\mathrm{m}} / 2 \pi$ for the $(1,2)$ mode from room temperature to $1.4 \mathrm{~K}$. Starting with $\gamma_{\mathrm{m}} / 2 \pi$ near $10 \mathrm{kHz}$ (corresponding to $Q_{\mathrm{m}} \sim 11,500$ ) at room temperature, $\gamma_{\mathrm{m}}$ increases with decreasing temperature, peaks near a bath temperature of $T_{\text {bath }}=50 \mathrm{~K}$ and then decreases with decreasing temperature with a plateau at temperatures between 10 and $4 \mathrm{~K}$. At $T_{\text {bath }}=1.4 \mathrm{~K}, Q_{\mathrm{m}}$ recovers to 3,700. In an amorphous solid, ultrasonic attenuations can occur through thermally activated relaxation processes for $T_{\text {bath }}>10 \mathrm{~K}$ (ref. 29). Below $5 \mathrm{~K}$, interactions of phonons with two-level tunnelling defects become important ${ }^{30}$. The temperature dependence shown in Fig. 2c is in good agreement with extensive earlier studies, indicating that $Q_{\mathrm{m}}$ should recover to the room-temperature value when the temperature is lowered to a few hundred millikelvin (ref. 30). Figure 2c also shows a weak temperature dependence $(<1 \%)$ of $\omega_{\mathrm{m}} / 2 \pi$. The sign reversal in the slope of the temperature dependence reflects a sign change of the thermal expansion coefficient for fused silica near $100 \mathrm{~K}$ (ref. 31).

The spectrally integrated area of a mechanical resonance in the displacement power spectrum is proportional to the temperature of the mechanical mode. In the limit that temperature-dependent variations in $\omega_{\mathrm{m}}$ and $m_{\text {eff }}$ are negligible, relative changes in the spectrally integrated area provide a direct measure of relative changes in the effective temperature of the mechanical mode. Figure $2 \mathrm{~d}$ shows the spectrally integrated area for the $(1,2)$ mode as a function of $T_{\text {bath }}$ (after subtraction of the shotnoise-limited background). The integrated area exhibits a linear dependence on $T_{\text {bath }}$, in spite of the strong temperature dependence of $\gamma_{\mathrm{m}} / 2 \pi$ shown in Fig. $2 \mathrm{c}$, confirming that in the absence of dynamical backaction, the mechanical oscillator is in thermal equilibrium with the bath.

We have carried out resolved-sideband cooling on the $(1,2)$ mode at $T_{\text {bath }}=3.6 \mathrm{~K}$ with the frequency of the incident laser beam, $\omega / 2 \pi$, tuned to $\omega_{\mathrm{m}}$ below the relevant WGM frequency, $\omega_{0} / 2 \pi$, unless otherwise specified, as illustrated in Fig. 3a. A deformed sphere with $\omega_{\mathrm{m}} / \kappa=5.4, \omega_{\mathrm{m}} / 2 \pi=123.4 \mathrm{MHz}, \gamma_{\mathrm{m}} / 2 \pi=12.5 \mathrm{kHz}$ (corresponding to $Q_{\mathrm{m}} \sim 10,000$ ) and $d=25.5 \mu \mathrm{m}$ at room 
a

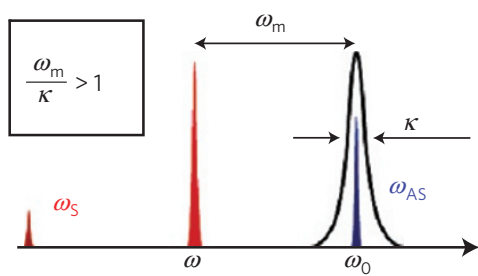

b

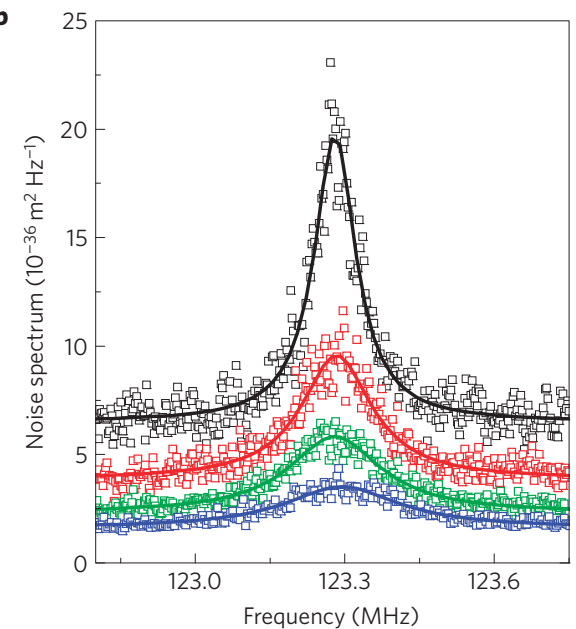

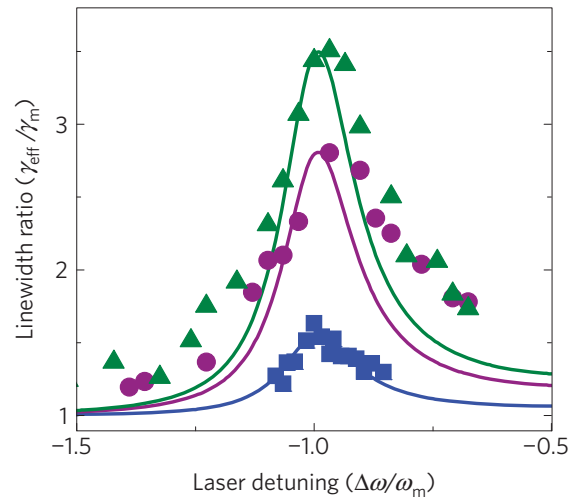

d

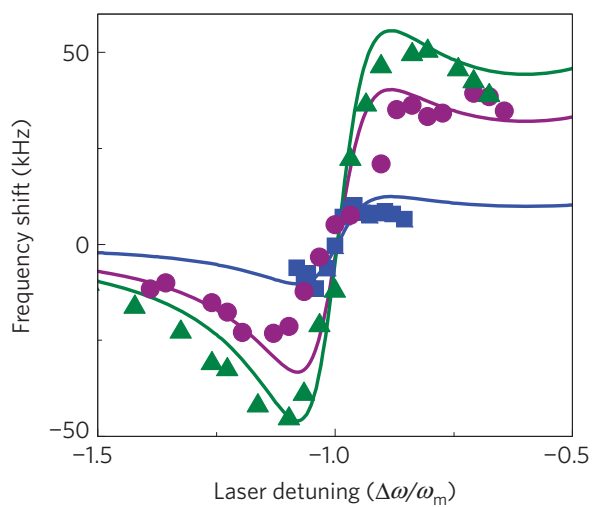

Figure 3 | Resolved-sideband cooling in a cryogenic environment. a, Schematic diagram of resolved-sideband cooling, where $\omega_{\mathrm{S}}$ ( $\omega_{\mathrm{AS}}$ ) denotes the frequency of Stokes (anti-Stokes) emission in the parametric process. $\mathbf{b}$, Displacement power spectrum obtained at $T_{\text {bath }}=3.6 \mathrm{~K}$ and $\Delta \omega / \omega_{\mathrm{m}}=-1$. The incident laser power is, from top to bottom, $10 \mathrm{~mW}, 40 \mathrm{~mW}, 60 \mathrm{~mW}$ and $83 \mathrm{~mW}$, respectively. The solid lines are Lorentzian fits. c,d, $\gamma_{\text {eff }} / \gamma_{\mathrm{m}}(\mathbf{c})$ and the mechanical frequency shift induced by optomechanical coupling (d) as a function of $\Delta \omega$ obtained at three incident laser powers: $20 \mathrm{~mW}$ (blue), $60 \mathrm{~mW}$ (purple) and $83 \mathrm{~mW}$ (olive). The solid lines are the results of the theoretical calculation discussed in the text.

temperature was precooled to $3.6 \mathrm{~K}$, at which point $\gamma_{\mathrm{m}} / 2 \pi=80 \mathrm{kHz}$ $\left(Q_{\mathrm{m}}=1,540\right)$. Figure $3 \mathrm{~b}$ shows the displacement power spectrum obtained at various incident laser powers. The spectrally integrated area of the mechanical resonance decreases with increasing laser power, accompanied by an increase in the effective damping rate of the mechanical mode. Note that although the incident laser power in these experiments can approach $100 \mathrm{~mW}$, the laser power coupled into the WGM is well below $50 \mu \mathrm{W}$ owing to the large laser detuning and the small free-space coupling efficiency (about a few per cent).

To characterize the resolved-sideband cooling process, we plot in Fig. 3c, d the ratio, $\gamma_{\text {eff }} / \gamma_{\mathrm{m}}=\left(\gamma_{\mathrm{m}}+\Gamma\right) / \gamma_{\mathrm{m}}$, where $\gamma_{\text {eff }}$ is the effective mechanical linewidth and $\Gamma$ is the radiationpressure cooling rate, and the mechanical frequency shift, $\Omega / 2 \pi$, induced by the optomechanical coupling as a function of the detuning, $\Delta \omega=\omega-\omega_{0}$. Theoretically, for resolved-sideband cooling we have ${ }^{18}$

$$
\begin{aligned}
\Gamma= & \gamma_{\mathrm{m}} \frac{P}{P_{\mathrm{th}}} \frac{4 \omega_{\mathrm{m}}^{2} \kappa}{4(\Delta \omega)^{2}+\kappa^{2}} \\
& \times\left[\frac{\kappa}{4\left(\Delta \omega+\omega_{\mathrm{m}}\right)^{2}+\kappa^{2}}-\frac{\kappa}{4\left(\Delta \omega-\omega_{\mathrm{m}}\right)^{2}+\kappa^{2}}\right] \\
\Omega= & \gamma_{\mathrm{m}} \frac{P}{P_{\mathrm{th}}} \frac{4 \omega_{\mathrm{m}}^{2} \kappa}{4(\Delta \omega)^{2}+\kappa^{2}} \\
& \times\left[\frac{\Delta \omega+\omega_{\mathrm{m}}}{4\left(\Delta \omega+\omega_{\mathrm{m}}\right)^{2}+\kappa^{2}}+\frac{\Delta \omega-\omega_{\mathrm{m}}}{4\left(\Delta \omega-\omega_{\mathrm{m}}\right)^{2}+\kappa^{2}}\right]
\end{aligned}
$$

where $P$ is the incident laser power and $P_{\text {th }}$ is the threshold incident power for parametric instability when $\Delta \omega=\omega_{\mathrm{m}}$. As shown in Fig. 3c, d, the observed $\Gamma$ and $\Omega$ are in good agreement with the calculation, for which $P_{\text {th }}=35 \mathrm{~mW}$, determined in a separate experiment, is used and there are no adjustable parameters. Note that large deviations between the experiment and calculation occur when the laser is tuned to near the WGM resonance (not shown). In this case, heating arising from optical absorption of the circulating light in silica becomes important and optical bistability can also occur $^{31}$. It should be added that at $T_{\text {bath }}<5 \mathrm{~K}$, no bistability is observed with $\Delta \omega / \omega_{\mathrm{m}}=-1$.

As we discussed earlier, a reliable measure of the effective temperature of a mechanical mode is the spectrally integrated area of the mechanical resonance in the displacement power spectrum. The spectrally integrated area includes contributions from all heating mechanisms, including optical absorption in silica and fluctuations of the laser or the WGM frequency. Figure $4 \mathrm{a}$ shows the spectrally integrated area, derived from the Lorentzian curve fitting such as those shown in Fig. 3b, as a function of the incident laser power. At $T_{\text {bath }}=3.6 \mathrm{~K}$ and $P=83 \mathrm{~mW}$, radiation-pressure cooling leads to a reduction in the area by factor of 3.5, indicating $T_{\text {eff }} \sim 1.0 \mathrm{~K}$ and an average final phonon occupation $\langle N\rangle \sim 170$. In the limit that optomechanical coupling induces no extra heating, the ratio $\gamma_{\text {eff }} / \gamma_{\mathrm{m}}$ can also be used to determine the effective temperature, with $T_{\text {eff }}=\left(\gamma_{\mathrm{m}} / \gamma_{\text {eff }}\right) T_{\text {bath }}$. The inset of Fig. 4a shows $\gamma_{\text {eff }} / 2 \pi$ as a function of the incident laser power, yielding a maximum cooling rate of $\Gamma / 2 \pi=200 \mathrm{kHz}$. The solid line in Fig. $4 \mathrm{a}$ is the calculated area using the slope obtained from the linear power dependence in the inset of Fig. 4a. The excellent agreement between $T_{\text {eff }}$ derived from the spectrally 

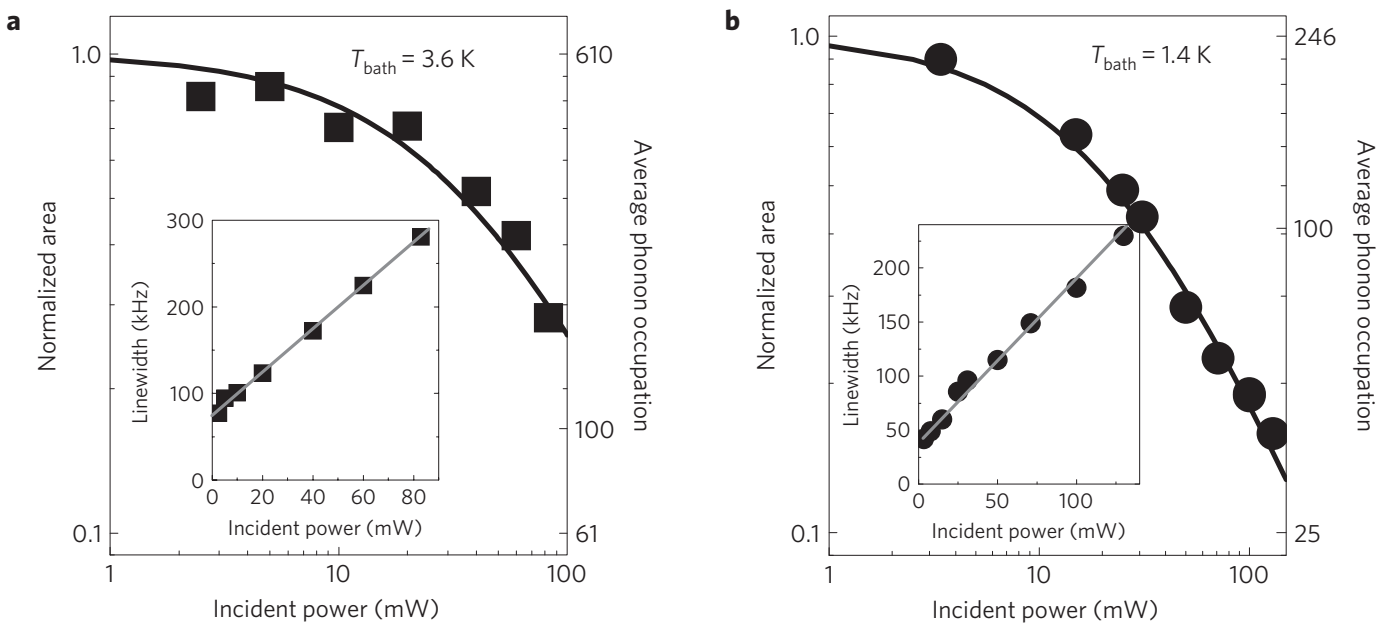

Figure 4 | Average phonon occupation. a,b, Spectrally integrated area of the $(1,2)$ mode obtained at different bath temperatures from the displacement noise spectrum and the corresponding effective mechanical linewidth (insets) as a function of the incident laser power with $\Delta \omega / \omega_{\mathrm{m}}=-1$. The area is normalized to that when the incident laser power approaches zero. The solid curves are the calculated area using the slope of the linear power dependence obtained from the inset.

integrated area and that derived from $\gamma_{\text {eff }} / \gamma_{\mathrm{m}}$ shows that under these experimental conditions, the incident laser beam induces negligible heating.

Resolved-sideband cooling at $T_{\text {bath }}=3.6 \mathrm{~K}$ is limited by the relatively large $\gamma_{\mathrm{m}}$ due to ultrasonic attenuation in silica, which should diminish at lower temperature. Within the limit of a ${ }^{4} \mathrm{He}$ cryostat, we have carried out resolved-sideband cooling for the $(1,2)$ mode at $T_{\text {bath }}=1.4 \mathrm{~K}$, for which a deformed microsphere with $\omega_{\mathrm{m}} / \kappa=4.0, \omega_{\mathrm{m}} / 2 \pi=118.6 \mathrm{MHz}$, $\gamma_{\mathrm{m}} / 2 \pi=11.5 \mathrm{kHz}$ and $d=26.5 \mu \mathrm{m}$ at room temperature was used. At $T_{\text {bath }}=1.4 \mathrm{~K}, \gamma_{\mathrm{m}} / 2 \pi=35 \mathrm{kHz}\left(Q_{\mathrm{m}}=3,400\right)$. Figure $4 \mathrm{~b}$ and its inset show the spectrally integrated area and the effective mechanical linewidth, derived from the displacement power spectra, as a function of the incident laser power. A maximum cooling rate of $\Gamma / 2 \pi=195 \mathrm{kHz}$, similar to that shown in the inset of Fig. $4 \mathrm{a}$, is obtained. As a result of the smaller $\gamma_{\mathrm{m}}$, this cooling rate leads to a reduction of the temperature for the mechanical mode by a factor of 6.6 , with $T_{\text {eff }} \sim 210 \mathrm{mK}$ and $\langle N\rangle \sim 37$. Figure $4 \mathrm{~b}$ also shows the excellent agreement between $T_{\text {eff }}$ derived from the spectrally integrated area and that derived from $\gamma_{\text {eff }} / \gamma_{\mathrm{m}}$, again indicating that the incident laser beam induces negligible heating.

Dynamical backaction cooling in optomechanical systems with high optical finesse is less susceptible to radiation-induced heating than that in electromechanical systems. Although slightly lower average phonon occupation $(\langle N\rangle \sim 25)$ has been achieved in cryogenically cooled electromechanical systems ${ }^{24}$, dynamical backaction cooling in these systems has been limited by heating arising from microwave radiation ${ }^{25}$. Average phonon occupation obtained with combined cryogenic and dynamical backaction cooling in electromechanical systems is considerably higher $(\langle N\rangle \sim 140)$.

In summary, using a deformed silica microsphere, we have reached a final average phonon occupation as low as 37 quanta by combining resolved-sideband cooling with cryogenic precooling. Although resolved-sideband cooling carried out at $T_{\text {bath }}=1.4 \mathrm{~K}$ is still limited by residual ultrasonic attenuation in silica, no special technical difficulty is anticipated in further lowering the precooling temperature, with a ${ }^{3} \mathrm{He}$ cryostat, to a few hundred millikelvin, at which effects of ultrasonic attention should diminish. The experimental results reported here thus indicate that we are tantalizingly close to realizing the groundstate cooling and reaching the quantum limit of a macroscopic optomechanical system.

\section{Methods}

Deformed silica microspheres were fabricated by fusing together two regular microspheres of similar sizes with a $\mathrm{CO}_{2}$ laser. The degree of deformation, defined as $\varepsilon=r_{a} / r_{b}-1$, where $r_{a}$ and $r_{b}$ are the radius of the long and short axis, respectively, can be gradually reduced through repeated heating. Silica microspheres used for optomechanical studies all feature a deformation less than $2 \%$. A Ti:sapphire ring laser frequency-stabilized to an external resonator near $\lambda=800 \mathrm{~nm}$ was used for free-space excitation of WGMs. For homodyne detection of the noise power spectrum, care has been taken to attenuate the input to the silicon photodiode such that the photodetector and the amplifier operate in the linear regime. For cryogenic precooling, a microsphere in an optical cryostat was placed in direct contact with either static exchange helium gas or vapours from a pumped helium reservoir. Further experimental details are presented in Supplementary Information.

Received 20 March 2009; accepted 11 May 2009; published online 7 June 2009

\section{References}

1. Schwab, K. C. \& Roukes, M. L. Putting mechanics into quantum mechanics. Phys. Today 58, 36-42 (2005).

2. Braginsky, V. B. \& Khalili, F. Y. Quantum Measurement (Cambridge Univ. Press, 1992).

3. Fabre, C., Pinard, M., Bourzeix, S., Heidmann, A., Giacobino, E. \& Reynaud, S. Quantum-noise reduction using a cavity with a movable mirror. Phys. Rev. A. 49, 1337-1343 (1994).

4. Bose, S., Jacobs, K. \& Knight, P. L. Scheme to probe the decoherence of a macroscopic object. Phys. Rev. A. 59, 3204-3210 (1999).

5. Mancini, S., Giovannetti, V., Vitali, D. \& Tombesi, P. Entangling macroscopic oscillators exploiting radiation pressure. Phys. Rev. Lett. 88, 120401 (2002).

6. Marshall, W., Simon, C., Penrose, R. \& Bouwmeester, D. Towards quantum superpositions of a mirror. Phys. Rev. Lett. 91, 130401 (2003).

7. Vitali, D. et al. Optomechanical entanglement between a movable mirror and a cavity field. Phys. Rev. Lett. 98, 030405 (2007).

8. Diedrich, F., Bergquist, J. C., Itano, W. M. \& Wineland, D. J. Laser cooling to the zero-point energy of motion. Phys. Rev. Lett. 62, 403-406 (1989).

9. Wilson-Rae, I., Nooshi, N., Zwerger, W. \& Kippenberg, T. J. Theory of ground state cooling of a mechanical oscillator using dynamical backaction. Phys. Rev. Lett. 99, 093901 (2007).

10. Marquardt, F., Chen, J. P., Clerk, A. A. \& Girvin, S. M. Quantum theory of cavity-assisted sideband cooling of mechanical motion. Phys. Rev. Lett. 99, 093902 (2007).

11. Schliesser, A., Riviere, R., Anetsberger, G., Arcizet, O. \& Kippenberg, T. J. Resolved-sideband cooling of a micromechanical oscillator. Nature Phys. 4 , 415-419 (2008).

12. Lacey, S., Wang, H., Foster, D. H. \& Nockel, J. U. Directional tunneling escape from nearly spherical optical resonators. Phys. Rev. Lett. 91, 033902 (2003).

13. Park, Y.-S., Cook, A. K. \& Wang, H. Cavity QED with diamond nanocrystals and silica microspheres. Nano. Lett. 6, 2075-2079 (2006).

14. Braginsky, V. B. Measurement of Weak Forces in Physics Experiments (Univ. Chicago Press, 1977). 
15. Kippenberg, T. J. \& Vahala, K. J. Cavity optomechanics: Back-action at the mesoscale. Science 321, 1172-1176 (2008).

16. Arcizet, O., Cohadon, P.-F., Briant, T., Pinard, M. \& Heidmann, A. Radiation-pressure cooling and optomechanical instability of a micromirror. Nature 444, 71-74 (2006).

17. Gigan, S. et al. Self-cooling of a micromirror by radiation pressure. Nature $\mathbf{4 4 4}$ 67-70 (2006)

18. Schliesser, A., Del'Haye, P., Nooshi, N., Vahala, K. J. \& Kippenberg, T. J. Radiation pressure cooling of a micromechanical oscillator using dynamical backaction. Phys. Rev. Lett. 97, 243905 (2006).

19. Ma, R. et al. Radiation-pressure-driven vibrational modes in ultrahigh-Q silica microspheres. Opt. Lett. 32, 2200-2202 (2007).

20. Thomson, J. D. et al. Strong dispersive coupling of a high-finesse cavity to a micromechanical membrane. Nature 452, 72-75 (2008).

21. Cohadon, P. F., Heidmann, A. \& Pinard, M. Cooling of a mirror by radiation pressure. Phys. Rev. Lett. 83, 3174-3177 (1999).

22. Kleckner, D. \& Bouwmeester, D. Sub-kelvin optical cooling of a micromechanical resonator. Nature 444, 75-78 (2006).

23. Groblacher, S., Gigan, S., Bohm, H. R., Zeilinger, A. \& Aspelmeyer, M. Radiation-pressure self-cooling of a micromirror in a cryogenic environment. Europhys. Lett. 81, 54003 (2008).

24. Naik, A. et al. Cooling a nanomechanical resonator with quantum back-action. Nature 443, 193-196 (2006).

25. Teufel, J. D., Harlow, J. W., Regal, C. A. \& Lehnert, K. W. Dynamical backaction of microwave fields on a nanomechanical oscillator. Phys. Rev. Lett. 101, 197203 (2008).
26. Braginsky, V. B., Gorodetsky, M. L. \& Ilchenko, V. S. Quality-factor and nonlinear properties of optical whispering-gallery modes. Phys. Lett. A 137, 393-397 (1989).

27. Vahala, K. J. Optical microcavities. Nature 424, 839-846 (2003).

28. Arcizet, O. et al. High-sensitivity optical monitoring of a micromechanical resonator with a quantum-limited optomechanical sensor. Phys. Rev. Lett. 97, 133601 (2006).

29. Vacher, R., Courtens, E. \& Foret, M. Anharmonic versus relaxational sound damping in glasses. 2. Vitreous silica. Phys. Rev. B 72, 214205 (2005).

30. Pohl, R. O., Liu, X. \& Thompson, E. Low-temperature thermal conductivity and acoustic attenuation in amorphous solids. Rev. Mod. Phys. 74, 991-1013 (2002).

31. Park, Y.-S. \& Wang, H. Regenerative pulsation in silica microspheres. Opt. Lett. 32, 3104-3106 (2007).

\section{Acknowledgements}

This work is supported by NSF and ARL-ONAMI.

\section{Author contributions}

Y.-S.P. and H.W. conceived the experiment, analysed the experimental result and prepared the manuscript. Y.-S.P. carried out the experiment.

\section{Additional information}

Supplementary information accompanies this paper on www.nature.com/naturephysics. Reprints and permissions information is available online at http://npg.nature.com/ reprintsandpermissions. Correspondence and requests for materials should be addressed to H.W. 